Pacific

Journal of

Mathematics

NONRATIONALITY OF NODAL QUARTIC THREEFOLDS

KYUSIK HONG 


\title{
NONRATIONALITY OF NODAL QUARTIC THREEFOLDS
}

\author{
KYUSIK HONG
}

\begin{abstract}
We prove the factoriality of every nodal quartic threefold with 13 singular points that contains neither planes nor quadric surfaces. As a corollary, any nodal quartic threefold with 13 singular points that contains neither planes nor quadric surfaces is nonrational.
\end{abstract}

\section{Introduction}

All varieties are assumed to be projective, normal, and defined over $\mathbb{C}$. A nodal variety is one that has, at most, isolated ordinary double points (nodes). A variety $V$ is said to be factorial if each Weil divisor is Cartier, and $\mathbb{Q}$-factorial if a multiple of each Weil divisor of $V$ is Cartier. This simple-looking definition is quite subtle when applied to projective varieties. It depends both on the kind of singularities and on their position. In the case of a Fano threefold $X, \mathbb{Q}$-factoriality is equivalent to the condition $\operatorname{rank}\left(H^{2}(X, \mathbb{Z})\right)=\operatorname{rank}\left(H_{4}(X, \mathbb{Z})\right)$. Thus a smooth Fano threefold is always $\mathbb{Q}$-factorial. The local class group at a node in a threefold has no torsion [Milnor 1968], so each Weil divisor that is $\mathbb{Q}$-Cartier must be a Cartier divisor on a nodal hypersurface in $\mathbb{P}^{4}$. Therefore, $\mathbb{Q}$-factoriality is equivalent to factoriality for a nodal hypersurface in $\mathbb{P}^{4}$. Moreover, the factoriality of a nodal quartic threefold implies its nonrationality; Mella [2004] proved that every factorial nodal quartic threefold is nonrational. This generalizes a classical result by Iskovskikh and Manin [1971] that every smooth quartic threefold is nonrational. On the other hand, there exist nonfactorial nodal quartic threefolds that are nonrational.

In view of Mella's result and the importance of rationality, studying the factoriality of nodal quartic threefolds is of interest. Here we consider this problem when the number of nodes is 13 . This extends earlier results, which we now quote.

Throughout, $X_{4}$ will represent a nodal quartic threefold.

Theorem 1.1 [Cheltsov 2006]. A quartic $X_{4}$ with at most 9 nodes is factorial if it contains no plane.

MSC2010: 14C20, 14J17, 14J30.

Keywords: factoriality, rationality, nodal quartic threefold. 
Theorem 1.2 [Shramov 2007, Theorem 1.3]. A quartic $X_{4}$ with at most 11 nodes is factorial if it contains no planes. If $X_{4}$ has 12 nodes, then $X_{4}$ is factorial, with the exception of the case when $X_{4}$ contains a quadric surface.

In Section 3 of this paper, we prove the following results.

Theorem 1.3. A quartic $X_{4}$ with at most 13 nodes is factorial if it contains neither planes nor quadric surfaces.

Corollary 1.4. A quartic $X_{4}$ with at most 13 nodes is nonrational if it contains neither planes nor quadric surfaces.

Theorem 1.3 improves on the degree-4 case of [Cheltsov 2006, Conjecture 13], which generalizes a well-known conjecture by Ciliberto [2004].

We present an example which motivates our study.

Example 1.5 [Cheltsov 2006, Example 10]. Let $a_{2}, h_{2}, b_{3}$ and $g_{1}$ be homogeneous polynomials of degrees $2,2,3$ and 1 , respectively. Consider the quartic threefold $X_{4}$ defined by the equation

$$
a_{2}(x, y, z, w, t) h_{2}(x, y, z, w, t)=b_{3}(x, y, z, w, t) g_{1}(x, y, z, w, t) ;
$$

it is the general quartic threefold passing through the quadric surface $Q$ defined by $a_{2}=g_{1}=0$. The quartic $X_{4}$ has 12 nodes, given by $h_{2}=g_{1}=a_{2}=b_{3}=0$, and it is not factorial.

\section{Preliminaries}

If the nodes of a nodal quartic threefold $X_{4}$ impose independent linear conditions on hypersurfaces of degree 3 in $\mathbb{P}^{4}$, then $X_{4}$ is factorial [Cynk 2001]. Hence, if a nodal quartic threefold $X_{4}$ is factorial, it must have at most 35 simple double points because $h^{0}\left(\mathbb{P}^{4}, \mathcal{O}_{\mathbb{P}^{4}}(3)\right)=35$. A nodal quartic threefold $X_{4}$ cannot have more than 45 nodes [Friedman 1986; Varchenko 1983]. Moreover, there is a unique nodal quartic threefold with 45 nodes [de Jong et al. 1990]. It is known as the Burkhardt quartic, which has too many nodes to be factorial.

The following result is one of the main tools.

Theorem 2.1 [Eisenbud and Koh 1989, Theorem 2]. Let $\Sigma$ be a set of points in $\mathbb{P}^{N}$ and let $d \geq 2$ be an integer. If no $d k+2$ of the points of $\Sigma$ lie in a projective $k$-plane for all $k \geq 1$, then $\Sigma$ imposes independent conditions on forms of degree d in $\mathbb{P}^{N}$.

We see that the singular points of nodal threefolds are located in $\mathbb{P}^{4}$ with the following properties:

Lemma 2.2 [Cheltsov and Park 2006, Lemma 2.9]. Let $X_{d}$ be a nodal hypersurface of degree d in $\mathbb{P}^{4}$. 
(1) A curve of degree $k$ in $\mathbb{P}^{4}$ contains at most $k(d-1)$ nodes of $X_{d}$.

(2) If a 2-plane contains $d(d-1) / 2+1$ nodes of $X_{d}$, then the 2-plane is contained in $X_{d}$.

More generally, a nodal hypersurface $X_{d}$ of degree $d$ in $\mathbb{P}^{4}$ is factorial if and only if the singular points of $X_{d}$ impose linearly independent conditions on hypersurfaces of degree $2 d-5$ in $\mathbb{P}^{4}$ [Cynk 2001]. To prove the factoriality of $X_{d}$, we have to compute whether $h^{1}\left(\mathbb{P}^{4}, \mathcal{O}_{\mathbb{P}^{4}}(2 d-5) \otimes \mathscr{I}_{\operatorname{Sing}\left(X_{d}\right)}\right)$ is 0 or not. Therefore, we need to study the dimension of the linear system $\left|\mathbb{O}_{\mathbb{p}^{4}}(2 d-5)\right|$ having assigned base points.

Fixing a nodal hypersurface $X_{d} \subset \mathbb{P}^{4}$ and $r$ singular points of $X_{d}$ in $\mathbb{P}^{4}$, what is the dimension of the space of hypersurfaces of degree $2 d-5$ in $\mathbb{P}^{4}$ passing through those points?

Let $\phi: \tilde{\mathbb{P}}^{4} \rightarrow \mathbb{P}^{4}$ be the blowing up of $\mathbb{P}^{4}$ along $\operatorname{Sing}\left(X_{d}\right)=\left\{p_{1}, \ldots, p_{r}\right\}$. Let $\tilde{X}_{d}$ be the strict transform of $X_{d}$, let $H$ be the divisor class of the pullback of a hyperplane under $\phi$, and let $E=\sum_{i=1}^{r} E_{i}$, where the $E_{i}$ are the classes of exceptional divisors. Suppose that $\mathscr{F} \in \mathrm{Pic} \mathbb{P}^{4}$ and $\mathscr{F}^{\prime}=\phi^{*} \mathscr{F}$. Then $\phi_{*} \mathrm{O}_{\tilde{\mathbb{P}}^{4}} \cong \mathrm{O}_{\mathbb{P}^{4}}$ and $R^{i} \phi_{*} \mathscr{F}^{\prime}=0$ for $i>0$. Therefore, $H^{j}\left(\mathbb{P}^{4}, \mathscr{F}\right) \cong H^{j}\left(\tilde{\mathbb{P}}^{4}, \mathscr{F}^{\prime}\right)$ by the Leray spectral sequence. Moreover, we have the equalities

$$
\begin{array}{rlrl}
\phi_{*}\left(\mathcal{O}_{\tilde{\mathbb{P}}^{4}}(-E)\right)=\Phi_{\operatorname{Sing}\left(X_{d}\right)}, & R^{i} \phi_{*}\left(\mathcal{O}_{\tilde{\mathbb{P}}^{4}}(-E)\right)=0 & \text { for } i>0, \\
\phi_{*}\left(\mathbb{O}_{\tilde{\mathbb{P}}^{4}}(k E)\right)=\mathbb{O}_{\mathbb{P}^{4}}, & R^{i} \phi_{*}\left(\mathbb{O}_{\tilde{\mathbb{P}}^{4}}(k E)\right)=0 \text { for } i>0, k=0,1,2,3 .
\end{array}
$$

By (2.3), we get $h^{j}\left(\tilde{\mathbb{P}}^{4}, \mathscr{O}_{\tilde{\mathbb{P}}^{4}}((2 d-5) H-E)\right)=h^{j}\left(\mathbb{P}^{4}, \mathscr{O}_{\mathbb{P}^{4}}(2 d-5) \otimes \mathscr{I}_{\operatorname{Sing}\left(X_{d}\right)}\right)$.

Let $L_{4}\left(2 d-5 ; 1^{r}\right)=L_{4}\left(2 d-5 ; 1_{1}, 1_{2}, \ldots, 1_{r}\right)$ be the complete linear system $\left|(2 d-5) H-\sum_{i=1}^{r} E_{i}\right|$ on $\tilde{\mathbb{P}}^{4}$. We will use the same notation to denote the corresponding line bundle on $\tilde{\mathbb{P}}^{4}$, as well as the push-forward of $\left|(2 d-5) H-\sum_{i=1}^{r} E_{i}\right|$ to $\mathbb{P}^{4}$, i.e., the linear system of threefolds of degree $2 d-5$ with multiplicity 1 at $p_{i}$.

Definition 2.5. A nonempty linear system $L_{4}\left(2 d-5 ; 1^{r}\right)$ is special if

$$
h^{0}\left(\tilde{\mathbb{P}}^{4}, L_{4}\left(2 d-5 ; 1^{r}\right)\right)>(2 d-1)(2 d-2)(2 d-3)(2 d-4) / 24-r
$$

or, which is the same, if $h^{1}\left(\tilde{\mathbb{P}}^{4}, L_{4}\left(2 d-5 ; 1^{r}\right)\right) \neq 0$.

Note that $h^{0}\left(\tilde{\mathbb{P}}^{4}, L_{4}\left(2 d-5 ; 1^{r}\right)\right) \geq h^{0}\left(\mathbb{P}^{4}, \mathcal{O}_{\mathbb{P}^{4}}(2 d-5)\right)-r$. We call the system $L_{4}\left(2 d-5 ; 1^{r}\right)$ nonspecial if $h^{0}\left(\tilde{\mathbb{P}}^{4}, L_{4}\left(2 d-5 ; 1^{r}\right)\right)=h^{0}\left(\mathbb{P}^{4}, \mathcal{O}_{\mathbb{P}^{4}}(2 d-5)\right)-r$, or, which is the same, if $h^{1}\left(\tilde{\mathbb{P}}^{4}, L_{4}\left(2 d-5 ; 1^{r}\right)\right)=0$.

Lemma 2.6. $h^{1}\left(\tilde{X}_{d}, O_{\tilde{X}_{d}}((2 d-5) H-E)\right)=0 \Leftrightarrow h^{1}\left(\tilde{\mathbb{P}}^{4}, O_{\tilde{\mathbb{P}}^{4}}((2 d-5) H-E)\right)=0$ Proof. Consider the exact sequence $0 \rightarrow \mathrm{O}_{\tilde{\mathbb{P}}^{4}}\left(-\tilde{X}_{d}+(2 d-5) H-E\right) \rightarrow \mathrm{O}_{\tilde{\mathbb{P}}^{4}}((2 d-5) H-E) \rightarrow \mathcal{O}_{\tilde{X}_{d}}((2 d-5) H-E) \rightarrow 0$. 
We have $\tilde{X}_{d} \equiv d H-2 E$. By (2.4),

$$
R^{i} \phi_{*}\left(\mathcal{O}_{\tilde{\mathbb{P}}^{4}}((d-5) H+E)\right)=\mathscr{O}_{\mathbb{P}^{4}}(d-5) \otimes R^{i} \phi_{*}\left(\mathscr{O}_{\tilde{\mathbb{P}}^{4}}(E)\right)=0
$$

for $i>0$. Then we get $h^{j}\left(\tilde{\mathbb{P}}^{4}, \mathscr{O}_{\tilde{\mathbb{p}} 3}((d-5) H+E)\right)=h^{j}\left(\mathbb{P}^{4}, \mathscr{O}_{\mathbb{P}^{4}}(d-5)\right)=0$ for $0<j<4$. Thus

$$
h^{1}\left(\tilde{X}_{d}, \mathcal{O}_{\tilde{X}_{d}}((2 d-5) H-E)\right)=h^{1}\left(\tilde{\mathbb{P}}^{4}, \mathcal{O}_{\tilde{\mathbb{P}}^{4}}((2 d-5) H-E)\right) .
$$

Therefore, studying linear systems of threefolds with assigned base points $p_{i}$ is equivalent to studying complete linear systems on the fourfold $\tilde{\mathbb{P}}^{4}$ obtained by blowing up the points $p_{i}$. Also, a nodal hypersurface $X_{d}$ of degree $d$ in $\mathbb{P}^{4}$ is factorial if and only if a nonempty linear system $L_{4}\left(2 d-5 ; 1^{r}\right)$ is nonspecial.

In the rest of this section we present tools to investigate the speciality of $L_{4}\left(3 ; 1^{r}\right)$ for $r \geq 12$. We need to consider the restriction on a quadric surface $Q$ due to Example 1.5 and Lemma 2.2.

Before stating these results, let $Q$ be a smooth quadric surface (when $Q$ is a singular quadric, we don't have a proof yet). Let $\operatorname{Sing}\left(X_{d}\right) \cap Q=\left\{p_{1}, p_{2}, \ldots, p_{\lambda}\right\}$, where $\lambda$ the maximal number of points of $\operatorname{Sing}\left(X_{d}\right)$ that can belong to the quadric $Q$. We consider a linear system $\left|\mathcal{O}\left(k_{1}, k_{2}\right) \otimes \mathscr{I}_{\operatorname{Sing}\left(X_{d}\right) \cap Q}\right|:=L_{Q}\left(\left(k_{1}, k_{2}\right) ; 1^{\lambda}\right)$ with $k_{1}>0$ and $k_{2}>0$ on the quadric $Q$ (that is, a system of curves of type $\left(k_{1}, k_{2}\right)$ through points $p_{i}$ of multiplicity 1 ). Using a similar method to Definition 2.5, we define the speciality for $L_{Q}\left(k_{1}, k_{2}\right)$. Then we see that

$$
h^{0}\left(Q, L_{Q}\left(\left(k_{1}, k_{2}\right) ; 1^{\lambda}\right)\right)=k_{1} k_{2}+k_{1}+k_{2}+1-\lambda
$$

if and only if the system $L_{Q}\left(\left(k_{1}, k_{2}\right) ; 1^{\lambda}\right)$ is nonspecial.

To prove the factoriality of a nodal quartic $X_{4}$, we have to investigate the speciality of the restriction system $\left.L_{4}\left(3 ; 1^{r}\right)\right|_{Q}=L_{Q}\left((3,3) ; 1^{\lambda}\right)$.

Lemma 2.7. With the above notation, let $\#\left|\operatorname{Sing}\left(X_{4}\right)\right|=r \geq 12$. Suppose the smooth quadric surface $Q$ is defined by $\left\{f_{2}(x, y, z, w, t)=0\right\} \cap A_{1}$, where $f_{2}$ is a homogeneous polynomial of degree 2 and $A_{1}$ is a hyperplane in $\mathbb{P}^{4}$ such that $\#\left|A_{1} \cap \operatorname{Sing}\left(X_{4}\right)\right| \geq 12$. Let $\lambda$ be the maximal number of points of $\operatorname{Sing}\left(X_{4}\right)$ that can belong to the smooth quadric $Q$.

(1) Suppose that $0 \leq r-\lambda \leq 2$. Then a linear system $L_{Q}\left((3,3) ; 1^{\lambda}\right)$ is special if and only if a linear system $L_{4}\left(3 ; 1^{r}\right)$ is special.

(2) Suppose that $3 \leq r-\lambda$. If a linear system $L_{Q}\left((3,3) ; 1^{\lambda}\right)$ is special, then a linear system $L_{4}\left(3 ; 1^{r}\right)$ is special.

Proof. Let $A_{1} \equiv H-\widehat{E}$ and let $\left\{f_{2}=0\right\} \equiv 2 H-\widehat{E}$, where $\widehat{E}=\sum_{i=1}^{\lambda} E_{i}$.

Consider the exact sequence

$$
\left.0 \rightarrow \mathfrak{O}_{\tilde{\mathbb{P}} 4}(-H+\widehat{E}+3 H-E) \rightarrow \mathfrak{O}_{\tilde{\mathbb{P}^{4}}}(3 H-E) \rightarrow \widehat{O}_{\tilde{\mathbb{P}} 4}(3 H-E)\right|_{A_{1}} \rightarrow 0 .
$$


We get the exact sequence

$$
\begin{aligned}
0 \rightarrow H^{0}\left(\mathrm{O}_{\tilde{\mathbb{p}}^{4}}\left(2 H-\sum_{i=\lambda+1}^{r} E_{i}\right)\right) \rightarrow H^{0}\left(\mathrm{O}_{\tilde{\mathbb{p}}^{4}}(3 H-E)\right) \\
\quad \rightarrow H^{0}\left(\left.\mathrm{O}_{\tilde{\mathbb{p}}^{4}}(3 H-E)\right|_{A_{1}}\right) \rightarrow H^{1}\left(\mathcal{O}_{\tilde{\mathbb{P}}^{4}}\left(2 H-\sum_{i=\lambda+1}^{r} E_{i}\right)\right) \\
\rightarrow H^{1}\left(\mathrm{O}_{\tilde{\mathbb{p}}^{4}}(3 H-E)\right) \rightarrow H^{1}\left(\left.\mathrm{O}_{\tilde{\mathbb{p}}^{4}}(3 H-E)\right|_{A_{1}}\right) \rightarrow 0 .
\end{aligned}
$$

Notice that $R^{j} \phi_{*} \widehat{O}_{\tilde{\mathbb{P}}^{4}}(2 H-E)=\mathcal{O}_{\mathbb{P}^{4}}(2) \otimes R^{j} \phi_{*} \widehat{O}_{\tilde{\mathbb{P}}^{4}}(-E)=0$ for all $j>0$. If $r=\lambda$, then $h^{1}\left(\mathcal{O}_{\tilde{\mathbb{P}}^{4}}\left(2 H-\sum_{i=\lambda+1}^{r} E_{i}\right)\right)=h^{1}\left(\mathcal{O}_{\mathbb{P}^{4}}(2)\right)=0$. Theorem 2.1 and Lemma 2.2(1) tell us that $h^{1}\left(\mathcal{O}_{\tilde{\mathbb{P}}^{4}}\left(2 H-\sum_{i=\lambda+1}^{r} E_{i}\right)\right)=h^{1}\left(\mathbb{P}^{4}, \mathscr{I}_{\sum_{i=\lambda+1}^{r} p_{i}}(2)\right)=0$ for $1 \leq r-\lambda \leq 5$. We then have $h^{1}\left(L_{4}\left(3 ; 1^{r}\right)\right)=h^{1}\left(\mathscr{O}_{\tilde{p}^{4}}(3 H-E)\right)=h^{1}\left(\left.\mathbb{O}_{\tilde{\mathbb{p}}^{4}}(3 H-E)\right|_{A_{1}}\right)$ when $0 \leq r-\lambda \leq 5$.

Also, note that $\left.Q \equiv(2 H-\widehat{E})\right|_{A_{1}}$. From the short exact sequence

$$
\left.0 \rightarrow \mathrm{O}_{A_{1}}(-2 H+\widehat{E}+3 H-E) \rightarrow \mathrm{O}_{A_{1}}(3 H-E) \rightarrow \mathfrak{O}_{A_{1}}(3 H-E)\right|_{Q} \rightarrow 0,
$$

we obtain the sequence

$$
\begin{aligned}
0 \rightarrow H^{0}\left(\mathcal{O}_{A_{1}}\left(H-\sum_{i=\lambda+1}^{r} E_{i}\right)\right) \rightarrow H^{0}\left(\mathbb{O}_{A_{1}}(3 H-E)\right) \\
\rightarrow H^{0}\left(\left.\mathbb{O}_{A_{1}}(3 H-E)\right|_{Q}\right) \rightarrow H^{1}\left(\mathcal{O}_{A_{1}}\left(H-\sum_{i=\lambda+1}^{r} E_{i}\right)\right) \\
\rightarrow H^{1}\left(\mathbb{O}_{A_{1}}(3 H-E)\right) \rightarrow H^{1}\left(\left.\mathbb{O}_{A_{1}}(3 H-E)\right|_{Q}\right) \rightarrow 0 .
\end{aligned}
$$

Note that $h^{1}\left(\mathcal{O}_{A_{1}}\left(H-\sum_{i=\lambda+1}^{r} E_{i}\right)\right)=h^{1}\left(\mathbb{P}^{3}, \Phi_{\sum_{i=\lambda+1}^{r} p_{i}}(1)\right)=0$ for $0 \leq r-\lambda \leq 2$. We have $h^{1}\left(\mathcal{O}_{A_{1}}(3 H-E)\right)=h^{1}\left(\left.\mathcal{O}_{A_{1}}(3 H-E)\right|_{Q}\right)=h^{1}\left(L_{Q}\left((3,3) ; 1^{\lambda}\right)\right)$ when $0 \leq r-\lambda \leq 2$.

The second statement follows from the last lines of (2.8) and (2.9).

Corollary 2.10. With the above notation, if $r=\lambda+j$ for $j=0,1,2$, then

$$
\begin{aligned}
& h^{0}\left(\mathcal{O}_{\tilde{\mathbb{P}}^{4}}(3 H-E)\right) \\
& \quad=h^{0}\left(L_{Q}\left((3,3) ; 1^{\lambda}\right)\right)+h^{0}\left(\mathcal{O}_{A_{1}}\left(H-\sum_{i=\lambda+1}^{r} E_{i}\right)\right)+h^{0}\left(\mathcal{O}_{\tilde{\mathbb{P}}^{4}}\left(2 H-\sum_{i=\lambda+1}^{r} E_{i}\right)\right) .
\end{aligned}
$$

Proof. This follows immediately from Lemma 2.7(1).

Lemma 2.11. Suppose that a curve of type $(a, b)$, with $0<a \leq b \leq 3$, and a curve of type $(3,3)$ meet in $3 a+3 b$ points, say $\Sigma_{(a, b)}=\left\{p_{1}, \ldots, p_{3 a+3 b}\right\}$.

(1) Let $\Psi \subset \Sigma_{(2,2)}$ and let $\alpha=\#|\Psi|$. Then a linear system $L_{Q}\left((3,3) ; 1^{\alpha}\right)$ is special if and only if $\alpha=12$, i.e., a curve of type $(2,2)$ contains $\Sigma_{(2,2)}$. 
(2) Let $\Omega \subset \Sigma_{(2,3)}\left(\right.$ or $\left.\Sigma_{(3,2)}\right)$ and let $\beta=\#|\Omega|$. Then a linear system $L_{Q}\left((3,3) ; 1^{\beta}\right)$ is special if and only if $\beta=14$.

(3) Let $\Upsilon \subset \Sigma_{(3,3)}$ and let $\gamma=\#|\Upsilon|$. Then a linear system $L_{Q}\left((3,3) ; 1^{\gamma}\right)$ is special if and only if $\gamma=15$.

Proof. By Lemma 2.2(1), a system $L_{Q}\left((3,3) ; 1^{\lambda}\right)$ has no fixed curve. The number $h_{\Sigma_{(a, b)}}(3,3)$ of conditions imposed by $\Sigma_{(a, b)}$ on forms of bidegree $(3,3)$ satisfies

$$
h_{\Sigma_{(a, b)}}(3,3)=h^{0}\left(L_{Q}(3,3)\right)-h^{0}\left(L_{Q}(3-a, 3-b)\right)-1 .
$$

There are four possible cases for the speciality of $L_{Q}\left((3,3) ; 1^{\lambda}\right)$.

When $(a, b)=(2,2)$, we get $h_{\Sigma_{(2,2)}}(3,3)=11<\#\left|\Sigma_{(2,2)}\right|$.

When $(a, b)=(2,3)($ or $(3,2))$, we can write $h_{\Sigma_{(2,3)}}(3,3)=13<\#\left|\Sigma_{(2,3)}\right|$ (or $\left.\#\left|\Sigma_{(3,2)}\right|\right)$, so the statement (2) is true.

Finally, the inequality $h_{\Sigma_{(3,3)}}(3,3)=14<\#\left|\Sigma_{(3,3)}\right|$ implies statement (3).

\section{The proof of Theorem 1.3}

Let $X_{4}$ be a nodal hypersurface in $\mathbb{P}^{4}$.

Definition 3.1. The set $\operatorname{Sing}\left(X_{4}\right)$ satisfies the property $\nabla$ if the following conditions hold:

- There is a hyperplane $A_{1}$ in $\mathbb{P}^{4}$ which contains at least 11 points of $\operatorname{Sing}\left(X_{4}\right)$.

- Fix an arbitrary point $p$ of $A_{1} \cap \operatorname{Sing}\left(X_{4}\right)$. There is a reducible cubic surface in $A_{1}$ passing through $\left(A_{1} \cap \operatorname{Sing}\left(X_{4}\right)\right) \backslash\{p\}$ but not passing through $p$.

Lemma 3.2. Let $\#\left|\operatorname{Sing}\left(X_{4}\right)\right|=11$. Suppose that there is a hyperplane $A_{1}$ in $\mathbb{P}^{4}$ such that $A_{1} \cap \operatorname{Sing}\left(X_{4}\right)=\operatorname{Sing}\left(X_{4}\right)$, and every quadric surface in $A_{1}$ does not contain all the points of $\operatorname{Sing}\left(X_{4}\right)$. Then $\operatorname{Sing}\left(X_{4}\right)$ satisfies the property $\nabla$.

Proof. Fix an arbitrary point $p$ of $\operatorname{Sing}\left(X_{4}\right)$. Let $\operatorname{Sing}\left(X_{4}\right)=\left\{p_{1}, p_{2}, \ldots, p_{10}, p\right\}$. Since every quadric surface does not contain all the points of $\operatorname{Sing}\left(X_{4}\right)$, we can find a quadric surface $Q_{1}$ in $A_{1}$ containing 9 points, say $\left\{p_{1}, p_{2}, \ldots, p_{8}, p_{9}\right\}$, of $\operatorname{Sing}\left(X_{4}\right) \backslash\{p\}$ but not containing $p$. We shall take for the required cubic surface the union of $Q_{1}$ and a two-dimensional linear subspace in $A_{1}$ passing through $p_{10}$ and not passing through $p$.

Lemma 3.3. Let $\mathcal{M} \subseteq\left|\mathbb{O}_{\mathbb{P}^{3}}(2)\right|$ be a linear subsystem that contains the set $A_{1} \cap$ $\operatorname{Sing}\left(X_{4}\right)$, where $A_{1}$ is a hyperplane in $\mathbb{P}^{4}$. Suppose that $n=\#\left|A_{1} \cap \operatorname{Sing}\left(X_{4}\right)\right| \geq 11$, $X_{4}$ contains no 2-planes, and a space curve of degree 4 in $A_{1}$ contains at most 10 points of $\operatorname{Sing}\left(X_{4}\right)$. Then the base locus $B s(\mathcal{M})$ is empty or two-dimensional. 
Proof. Suppose that $B s(\mathcal{M})$ is zero-dimensional. Let $M_{1}, M_{2}$, and $M_{3}$ be the general surfaces of $M$. Then the intersection number $M_{1} \cdot M_{2} \cdot M_{3}$ has at most 8 , but $n \geqslant 11$ holds.

Now we suppose that the curve $B=B s(\mathcal{M}) \subset A_{1}$. Then $\operatorname{deg} B \leq 4$. Since $n \geqslant 11$, by Lemma 2.2(1), $\operatorname{deg} B=4$, and $B$ must be reduced. Moreover, $B$ is not contained in a two-dimensional linear subspace, because a two-dimensional linear subspace contains at most 6 points. This contradicts the assumption.

Lemma 3.4. Let $\#\left|\operatorname{Sing}\left(X_{4}\right)\right|=11$. Suppose that $X_{4}$ contains no 2-planes, there is a hyperplane $A_{1}$ in $\mathbb{P}^{4}$ such that $A_{1} \cap \operatorname{Sing}\left(X_{4}\right)=\operatorname{Sing}\left(X_{4}\right)$, every reducible quadric surface does not contain all the points of $\operatorname{Sing}\left(X_{4}\right)$, and a space curve of degree 4 in $A_{1}$ does not pass through all the points of $\operatorname{Sing}\left(X_{4}\right)$. Then $\operatorname{Sing}\left(X_{4}\right)$ satisfies the property $\nabla$.

Proof. Fix an arbitrary point $p$ of $\operatorname{Sing}\left(X_{4}\right)$. Let $\operatorname{Sing}\left(X_{4}\right)=\left\{p_{1}, p_{2}, \ldots, p_{10}, p\right\}$. By Lemma 3.2, we assume that there is an irreducible quadric surface $Q_{2}$ in $A_{1}$ containing all the points of $\operatorname{Sing}\left(X_{4}\right)$. By Lemma 3.3, any quadric surface in $A_{1}$ passing through all the points of $\operatorname{Sing}\left(X_{4}\right)$ coincides with $Q_{2}$. Suppose that $Q_{2}$ is determined by 8 points, say $\left\{p_{1}, p_{2}, \ldots, p_{8}\right\}$, of $\operatorname{Sing}\left(X_{4}\right) \backslash\{p\}$ together with $p$. Then we can find a quadric $Q_{3}$ in $A_{1}$ containing $\left\{p_{1}, p_{2}, \ldots, p_{8}\right\}$ and not containing $p$. We can assume that $p_{k} \notin Q_{3}$ for $k=9$ or 10 ; otherwise, take a two-dimensional linear subspace in $A_{1}$ containing the point $\operatorname{Sing}\left(X_{4}\right) \backslash Q_{3}$ but not containing $p$.

If $p \notin \overline{p_{9}, p_{10}}$, then we can easily construct a reducible cubic surface in $A_{1}$ that contains $\operatorname{Sing}\left(X_{4}\right) \backslash\{p\}$ and does not contain $p$.

Now we suppose that three points $\left\{p_{9}, p_{10}, p\right\}$ lie on a single line. By statement (1) of Lemma 2.2, the line determined by $\left\{p_{i}, p_{9}\right\}$ (or $\left\{p_{i}, p_{10}\right\}$ ), for $1 \leq i \leq 8$, does not pass through $p$. We consider the quadric surface $Q_{4}$ determined by $\left\{p_{1}, p_{2}, \ldots, p_{8}, p_{9}\right\}$. We can assume that the quadric surface $Q_{4}$ contains the points $p$; otherwise, take a two-dimensional linear subspace in $A_{1}$ containing the point $p_{10}$ but not containing $p$.

Then $Q_{4}$ must be $Q_{2}$, that is, the quadric surface $Q_{2}$ is determined by the point $p$ and $\left\{p_{1}, p_{2}, \ldots, p_{8}, p_{9}\right\} \backslash\left\{p_{j}\right\}$ for $1 \leq j \leq 9$. Therefore, we can find a quadric surface $Q_{5}$ passing through $\left\{p_{1}, p_{2}, \ldots, p_{8}, p_{9}\right\} \backslash\left\{p_{j}\right\}$, for $1 \leq j \leq 8$ and not passing through $p$. Let $l$ be the line determined by two points $\operatorname{Sing}\left(X_{4}\right) \backslash Q_{5} \backslash\{p\}$. Then $p$ cannot lie on the line $l$. Let $\overline{A_{1}}$ be a two-dimensional linear subspace in $A_{1}$ containing the line $l$ but not containing $p$. Then the union of $Q_{5}$ and $\overline{A_{1}}$ is the desired form of degree 3 .

Lemma 3.5. Let $\#\left|\operatorname{Sing}\left(X_{4}\right)\right|=11$. Suppose that $X_{4}$ contains no 2-planes, there is a hyperplane $A_{1}$ in $\mathbb{P}^{4}$ such that $A_{1} \cap \operatorname{Sing}\left(X_{4}\right)=\operatorname{Sing}\left(X_{4}\right)$, every reducible quadric surface does not contain all the points of $\operatorname{Sing}\left(X_{4}\right)$, and there is a space 
curve $D$ of degree 4 in $A_{1}$ that passes through all the points of $\operatorname{Sing}\left(X_{4}\right)$. Then $\operatorname{Sing}\left(X_{4}\right)$ satisfies the property $\nabla$.

Proof. Fix an arbitrary point $p$ of $\operatorname{Sing}\left(X_{4}\right)$. Let $\operatorname{Sing}\left(X_{4}\right)=\left\{p_{1}, p_{2}, \ldots, p_{10}, p\right\}$. By Lemma 3.2, we assume that there is an irreducible quadric surface containing all the points of $\operatorname{Sing}\left(X_{4}\right)$. By Lemma 2.2(1), a twisted cubic contains at most 9 singular points of $X_{4}$. Lemma 3.3 tells us that $D$ can be written as antersection of two different quadric surfaces in $A_{1}$. Then there is a quadric surface in $A_{1}$ containing 7 points, say $\left\{p_{1}, \ldots, p_{7}\right\}$, of $\operatorname{Sing}\left(X_{4}\right) \backslash\{p\}$ but not containing $p$. We consider the two-dimensional linear subspace $\hat{A}_{1}$ in $A_{1}$ determined by $\left\{p_{8}, p_{9}, p_{10}\right\}$. We can assume that $p \in \hat{A}_{1}$; otherwise, one can easily construct the required cubic surface in $A_{1}$.

By Lemma 2.2(1), renumbering $p_{8}, p_{9}$, and $p_{10}$ if necessary, we can assume that $\left\{p_{9}, p_{10}, p\right\}$ span $\hat{A}_{1}$. By Lemma 2.2(2), $\hat{A}_{1}$ contains at most 2 points of $\left\{p_{1}, \ldots, p_{7}\right\}$. We can assume that $\hat{A}_{1}$ contains 2 points, say $\left\{p_{6}, p_{7}\right\}$, of $\left\{p_{1}, \ldots, p_{7}\right\}$ (a similar method applies to the case when $\hat{A}_{1}$ passes through one or none of $\left.\left\{p_{1}, \ldots, p_{7}\right\}\right)$. Assume that all the quadric surfaces in $A_{1}$ containing 7 points, $\left\{p_{1}, \ldots, p_{7}, p_{8}\right\} \backslash\left\{p_{i}\right\}$ for $1 \leq i \leq 5$, also pass through $p$. Then each quadric surface containing the points $p_{6}, p_{7}$, and $p_{8}$ also contains $p$, and hence $p_{6}, p_{7}, p_{8}$, and $p$ lie on a single line. This is a contradiction to Lemma 2.2(1). Thus, we can find a quadric surface $Q_{6}$ in $A_{1}$ containing 7 points, $\left\{p_{1}, \ldots, p_{7}, p_{8}\right\} \backslash\left\{p_{i}\right\}$ for $1 \leq i \leq 5$, and not containing $p$. Take the two-dimensional linear subspace $A_{1}^{\prime}$ in $A_{1}$ determined by three points $\operatorname{Sing}\left(X_{4}\right) \backslash Q_{6} \backslash\{p\}$. Then $A_{1}^{\prime}$ does not contain $p$, and hence $Q_{6}+A_{1}^{\prime}$ is the required cubic surface.

Proposition 3.6. Let $\#\left|\operatorname{Sing}\left(X_{4}\right)\right|=11$. Suppose that $X_{4}$ contains no 2-planes, a hyperplane in $\mathbb{P}^{4}$ contains all the points of $\operatorname{Sing}\left(X_{4}\right)$, and every reducible quadric surface does not contain all the points of $\operatorname{Sing}\left(X_{4}\right)$. Then $\operatorname{Sing}\left(X_{4}\right)$ satisfies the property $\nabla$.

Proof. By Lemma 3.2, we can assume that there is an irreducible quadric surface containing all the points of $\operatorname{Sing}\left(X_{4}\right)$. It immediately follows from Lemmas 3.4 and 3.5.

The following result is proved in [Shramov 2007].

Corollary 3.7 [Shramov 2007, Corollary 3.4]. Let $\operatorname{Sing}\left(X_{4}\right)=\left\{p_{1}, \ldots, p_{s}\right\}$. Assume that $s \geq 11$, and no 2-plane contains 7 points of $\operatorname{Sing}\left(X_{4}\right)$. Let $p_{1}, \ldots, p_{s}$ be points lying in a reducible quadric surface (that is, in a pair of planes spanning a three-dimensional surface). Then either $p_{1}, \ldots, p_{s}$ impose independent conditions on the forms of degree 3 or $p_{1}, \ldots, p_{s}$ also lie in an irreducible quadric surface.

Proposition 3.8. Let \#|Sing $\left(X_{4}\right) \mid=13$. Suppose that $X_{4}$ contains no 2-planes, and a hyperplane in $\mathbb{P}^{4}$ contains at most 11 points of $\operatorname{Sing}\left(X_{4}\right)$. Then $X_{4}$ is factorial. 
Proof. Fix an arbitrary point $p$ of $\operatorname{Sing}\left(X_{4}\right)$. It is enough to construct a cubic threefold $T$ that contains $\operatorname{Sing}\left(X_{4}\right) \backslash\{p\}$ and does not contain $p$.

Suppose that there is a hyperplane $A_{1}$ in $\mathbb{P}^{4}$ containing 11 points of $\operatorname{Sing}\left(X_{4}\right)$; otherwise, $X_{4}$ is factorial due to Theorem 2.1 and Lemma 2.2.

Suppose that $p \notin A_{1}$. We can find a quadric threefold $G$ passing through two points $\operatorname{Sing}\left(X_{4}\right) \backslash A_{1} \backslash\{p\}$ but not passing through $p$. Then $T=A_{1}+G$.

Now assume that $p \in A_{1}$. We divide the case into two subcases. Let $\left\{q_{1}, q_{2}\right\}=$ $\operatorname{Sing}\left(X_{4}\right) \backslash A_{1}$.

First, assume that a reducible quadric surface in $A_{1}$ does not contain all the points of $\operatorname{Sing}\left(X_{4}\right)$. By Proposition 3.6, we obtain a cubic surface $W=Q^{\prime}+\Lambda$, where $Q^{\prime}$ is a quadric surface and $\Lambda$ is a two-dimensional linear subspace, in $A_{1}$ containing $\operatorname{Sing}\left(X_{4}\right) \cap\left(A_{1} \backslash\{p\}\right)$ but not containing $p$. Take the cone $Q^{\prime \prime}$ over $Q^{\prime}$ with vertex $q_{1}$ and a hyperplane $\Lambda^{\prime}$ in $\mathbb{P}^{4}$ containing $\Lambda$ together with $q_{2}$. Then we can get the required cubic threefold $T$ as the union of $Q^{\prime \prime}$ and $\Lambda^{\prime}$.

Second, assume that a reducible quadric surface in $A_{1}$ contains all the points of $\operatorname{Sing}\left(X_{4}\right)$. Applying the proof of Corollary 3.7, we can construct a reducible cubic surface $Y$ in $A_{1}$ passing through $\left(\operatorname{Sing}\left(X_{4}\right) \cap A_{1}\right) \backslash\{p\}$ and not passing through $p$ such that $Y$ consists of three two-dimensional linear subspaces, say $L_{1}, L_{2}$, and $L_{3}$. Note that $q_{1}, q_{2} \notin A_{1}$. Then we obtain the required cubic threefold $T$ as the union of a hyperplane in $\mathbb{P}^{4}$ containing $\left\{L_{1}, q_{1}\right\}$, a hyperplane in $\mathbb{P}^{4}$ containing $\left\{L_{2}, q_{2}\right\}$, and a hyperplane in $\mathbb{P}^{4}$ containing $L_{3}$ but not containing $p$.

The following three results are proved in [Shramov 2007]. They are very useful for the proof of Theorem 1.3. We let $\operatorname{Sing}\left(X_{4}\right)=\left\{p_{1}, \ldots, p_{s}\right\}$.

Lemma 3.9 [Shramov 2007, Lemma 3.5]. Assume that $s \leq 12$, and no 2-plane contains 7 points of $\operatorname{Sing}\left(X_{4}\right)$. Let $p_{1}, \ldots, p_{s}$ be points in a 3-dimensional subspace $\mathbb{P}^{3} \subset \mathbb{P}^{4}$ not lying in some quadric surface. Then $p_{1}, \ldots, p_{s}$ impose independent conditions on the forms of degree 3 in $\mathbb{P}^{3}$ (and therefore also in $\mathbb{P}^{4}$ ).

Lemma 3.10 [Shramov 2007, Lemma 3.8]. Assume that no 2-plane contains 7 points of $\operatorname{Sing}\left(X_{4}\right)$. Let $p_{1}, \ldots, p_{12}$ be points in a quadric surface. Then either $p_{1}, \ldots, p_{12}$ impose independent conditions on the forms of degree 3 in $\mathbb{P}^{4}$ or $p_{1}, \ldots, p_{12}$ lie in a pencil of quadric surfaces in some 3-dimensional subspace.

Lemma 3.11 [Shramov 2007, Lemma 3.9]. Assume that no 2-plane contains 7 points of $\operatorname{Sing}\left(X_{4}\right)$. Let $p_{1}, \ldots, p_{12}$ be points lying in a pencil of quadric surfaces in a 3-dimensional subspace. Then $X_{4}$ contains a quadric surface.

Proposition 3.12. Let $\#\left|\operatorname{Sing}\left(X_{4}\right)\right|=13$. Suppose that $X_{4}$ contains no 2-planes, and a hyperplane in $\mathbb{P}^{4}$ contains at most 12 points of $\operatorname{Sing}\left(X_{4}\right)$. Then $X_{4}$ is either factorial or contains a quadric surface. 
Proof. Fix an arbitrary point $p$ of $\operatorname{Sing}\left(X_{4}\right)$. We can assume that there is a hyperplane $A_{1}$ in $\mathbb{P}^{4}$ containing 12 points of $\operatorname{Sing}\left(X_{4}\right)$. Let $\{q\}=\operatorname{Sing}\left(X_{4}\right) \backslash A_{1}$.

First, suppose that a quadric surface contains at most 11 points of $\operatorname{Sing}\left(X_{4}\right)$. We can assume that $p \in A_{1}$; otherwise, one can easily check that $X_{4}$ is factorial. By Lemma 3.9, we can find a cubic surface $U$ in $A_{1}$ passing through ( $\left.\operatorname{Sing}\left(X_{4}\right) \cap A_{1}\right) \backslash\{p\}$ and not passing through $p$. Taking a cone over $U$ with vertex $q$, we obtain a cubic threefold passing through $\operatorname{Sing}\left(X_{4}\right) \backslash\{p\}$ and not passing through $p$. In this case, $X_{4}$ is factorial.

Second, suppose that there is a quadric surface $\widehat{Q}$ containing 12 points, say $\Xi$, of $\operatorname{Sing}\left(X_{4}\right)$. We can assume that $\Xi$ cannot lie on a pencil of quadric surface in $A_{1}$; otherwise, by Lemma 3.11, $X_{4}$ contains a quadric surface.

Now we have to prove that $X_{4}$ is factorial. We can assume that $p \in \widehat{Q}$. Let $\Xi=\left\{p_{1}, \ldots, p_{11}, p\right\}$. Applying the proof of Lemma 3.10, we obtain a reducible cubic surface $K$ in $A_{1}$ containing $\Xi \backslash\{p\}$ but not containing $p$. Note that $q \notin A_{1}$. Let $K=S+L$, where $S$ is a quadric surface and $L$ is a two-dimensional linear subspace. Then we can construct a cubic threefold as the union of the cone over $S$ with vertex $q$ and a hyperplane in $\mathbb{P}^{4}$ containing $L$ but not containing $p$. Thus, $X_{4}$ is factorial.

Proposition 3.13. Let \#|Sing $\left(X_{4}\right) \mid=13$. Suppose that $X_{4}$ contains no 2-planes, and there is a hyperplane $A_{1}$ in $\mathbb{P}^{4}$ containing all the points of $\operatorname{Sing}\left(X_{4}\right)$. Then $X_{4}$ is either factorial or contains a quadric surface.

Proof. Fix an arbitrary point $p$ of $\operatorname{Sing}\left(X_{4}\right)$. Let $\operatorname{Sing}\left(X_{4}\right)=\left\{p_{1}, \ldots, p_{12}, p\right\}$.

Suppose that every quadric surface does not contain all the points of $\operatorname{Sing}\left(X_{4}\right)$. Then we find a quadric surface containing 9 points, say $\left\{p_{1}, \ldots, p_{9}\right\}$, of $\operatorname{Sing}\left(X_{4}\right)$ but not containing $p$. We consider the two-dimensional linear subspace $\hat{A}_{1}$ in $A_{1}$ determined by $\left\{p_{10}, p_{11}, p_{12}\right\}$. We can assume that $p \in \hat{A}_{1}$; otherwise, one can easily check that $X_{4}$ is factorial.

By Lemma 2.2(1), renumbering $p_{10}, p_{11}$, and $p_{12}$ if necessary, we can assume that $\left\{p_{11}, p_{12}, p\right\}$ span $\hat{A}_{1}$. By Lemma $2.2(2), \hat{A}_{1}$ contains at most 2 points of $\left\{p_{1}, \ldots, p_{9}\right\}$. We can assume that $\hat{A}_{1}$ contains 2 points, say $\left\{p_{8}, p_{9}\right\}$, of $\left\{p_{1}, \ldots, p_{9}\right\}$ (a similar method applies to the case when $\hat{A}_{1}$ passes through one or none of $\left.\left\{p_{1}, \ldots, p_{9}\right\}\right)$. Assume that all the quadric surfaces in $A_{1}$ containing 9 points, $\left\{p_{1}, \ldots, p_{9}, p_{10}\right\} \backslash\left\{p_{i}\right\}$ for $1 \leq i \leq 7$, also pass through $p$. Then each quadric surface containing the points $p_{8}, p_{9}$, and $p_{10}$ also contains $p$, and hence $p_{8}, p_{9}, p_{10}$, and $p$ lie on a single line. This is a contradiction to Lemma 2.2(1). Thus, we can find a quadric surface $Q_{7}$ in $A_{1}$ containing 9 points, $\left\{p_{1}, \ldots, p_{9}, p_{10}\right\} \backslash\left\{p_{i}\right\}$ for $1 \leq i \leq 7$, and not containing $p$. The union of $Q_{7}$ and the two-dimensional linear subspace $A_{1}^{\prime \prime}$ in $A_{1}$ determined by three points $\operatorname{Sing}\left(X_{4}\right) \backslash Q_{7} \backslash\{p\}$ is a cubic surface containing $\operatorname{Sing}\left(X_{4}\right) \backslash\{p\}$ but not containing $p$. It implies that $X_{4}$ is factorial. 
Now suppose that there is a quadric surface $Q_{8}$ containing all the points of $\operatorname{Sing}\left(X_{4}\right)$. Then, by Lemma 2.2(2), $Q_{8}$ is irreducible. We may assume that $Q_{8}$ is a quadric cone; otherwise, by Lemma 2.7(1) and 2.11(1), $X_{4}$ is either factorial or contains a quadric surface. For instance, since a curve of type $(1,1)$ contains at most 6 points of $\operatorname{Sing}\left(X_{4}\right), X_{4}$ cannot contain a 2-plane.

If there is a quadric surface different from $Q_{8}$ containing 12 points of $\operatorname{Sing}\left(X_{4}\right)$, then $X_{4}$ contains a quadric surface due to Lemma 3.11.

We can assume $Q_{8}$ is unique; that is, any quadric surface different from $Q_{8}$ passes through at most 11 points of $\operatorname{Sing}\left(X_{4}\right)$. Consider a nodal quartic threefold $\widehat{X}_{4}$ defined by

$$
a_{1}(x, y, z, t, w) h_{3}(x, y, z, t, w)+b_{2}(x, y, z, t, w) g_{2}(x, y, z, t, w)=0,
$$

where $a_{1}, h_{3}, b_{2}$ and $g_{2}$ are homogeneous polynomials of degree $1,3,2$, and 2, respectively. Suppose that the quadric $Q_{8}$ is the quadric cone given by $\left\{a_{1}=b_{2}=0\right\}$, and $V_{2}$ is a quadric surface given by $\left\{a_{1}=g_{2}=0\right\}$. Then the nodes of $\widehat{X}_{4}$ are $\left\{a_{1}=h_{3}=b_{2}=g_{2}=0\right\}$ and the vertex of $Q_{8}$. The quartic $\widehat{X}_{4}$ has 13 nodes with $\operatorname{Sing}\left(\widehat{X}_{4}\right)=\operatorname{Sing}\left(X_{4}\right)$, and all the points of $\operatorname{Sing}\left(\widehat{X}_{4}\right)$ lie on a hyperplane $\left\{a_{1}=0\right\}$. By the uniqueness of $Q_{8}, X_{4}$ must be $\widehat{X}_{4}$. Since $V_{2}$ contains 12 points of $\operatorname{Sing}\left(X_{4}\right)$, this contradicts the assumption.

Proof of Theorem 1.3. Suppose that every two dimensional linear subspace contains at most 6 singular points of a nodal quartic $X_{4}$, i.e, by Lemma 2.2(2), $X_{4}$ contains no 2-planes; otherwise, $X_{4}$ is defined by an equation of the form

$$
y_{1}(x, y, z, t, w) f_{3}(x, y, z, t, w)+\hat{y}_{1}(x, y, z, t, w) g_{3}(x, y, z, t, w)=0,
$$

where $y_{1}, f_{3}, \hat{y}_{1}$, and $g_{3}$ are homogeneous polynomials of degree $1,3,1$, and 3 , respectively. Then $X_{4}$ is not factorial.

Theorem 1.3 immediately follows from Propositions 3.8, 3.12, and 3.13.

\section{Acknowledgments}

The author would like to thank two referees for their valuable comments and suggestions. This work was supported by the National Research Foundation of Korea (NRF) grant funded by the Korean government (MEST) (No. 2011-0001565).

\section{References}

[Cheltsov 2006] I. Cheltsov, "Nonrational nodal quartic threefolds", Pacific J. Math. 226:1 (2006), 65-81. MR 2007e:14024 Zbl 1123.14010

[Cheltsov and Park 2006] I. Cheltsov and J. Park, "Factorial hypersurfaces in $\mathbb{P}^{4}$ with nodes", Geom. Dedicata 121 (2006), 205-219. MR 2007m:14064 Zbl 1110.14031 
[Ciliberto and Di Gennaro 2004] C. Ciliberto and V. Di Gennaro, "Factoriality of certain hypersurfaces of $\mathbb{P}^{4}$ with ordinary double points", pp. 1-7 in Algebraic transformation groups and algebraic varieties, edited by V. L. Popov, Encyclopaedia Math. Sci. 132, Springer, Berlin, 2004. MR 2005i:14043 Zbl 1068.14010

[Cynk 2001] S. Cynk, "Defect of a nodal hypersurface", Manuscripta Math. 104:3 (2001), 325-331. MR 2002g:14056 Zbl 0983.14017

[Eisenbud and Koh 1989] D. Eisenbud and J.-H. Koh, "Remarks on points in a projective space", pp. 157-172 in Commutative algebra (Berkeley, CA, 1987), edited by M. Hochster et al., Math. Sci. Res. Inst. Publ. 15, Springer, New York, 1989. MR 90i:14008 Zbl 0736.14022

[Friedman 1986] R. Friedman, "Simultaneous resolution of threefold double points", Math. Ann. 274:4 (1986), 671-689. MR 87k:32035 Zbl 0576.14013

[Iskovskih and Manin 1971] V. A. Iskovskih and J. I. Manin, "Three-dimensional quartics and counterexamples to the Lüroth problem”, Mat. Sb. (N.S.) 86(128):1(9) (1971), 140-166. In Russian; translated in Math. USSR-Sb. 15:1 (1971), 141-166. MR 45 \#266 Zbl 0222.14009

[de Jong et al. 1990] A. J. de Jong, N. I. Shepherd-Barron, and A. Van de Ven, "On the Burkhardt quartic", Math. Ann. 286:1-3 (1990), 309-328. MR 91f:14038 Zbl 0712.14023

[Mella 2004] M. Mella, "Birational geometry of quartic 3-folds, II: The importance of being $\mathbb{Q}$ factorial”, Math. Ann. 330:1 (2004), 107-126. MR 2005h:14030 Zbl 1058.14022

[Milnor 1968] J. Milnor, Singular points of complex hypersurfaces, Annals of Mathematics Studies 61, Princeton University Press, 1968. MR 39 \#969 Zbl 0184.48405

[Shramov 2007] K. A. Shramov, “QQ-factorial quartic threefolds”, Mat. Sb. 198:7 (2007), 103-114. In Russian; translated in Sb. Math. 198:8 (2007), 1165-1174. MR 2008i:14062 Zbl 1132.14039

[Varchenko 1983] A. N. Varchenko, "Semicontinuity of the spectrum and an upper bound for the number of singular points of the projective hypersurface", Dokl. Akad. Nauk SSSR 270:6 (1983), 1294 1297. In Russian, translated in Sov. Math. Dokl. 27 (1983), 735-739. MR 85d:32028 Zbl 0537.14003

Received April 29, 2012. Revised November 14, 2012.

KYUSIK HONG

Department of Mathematics and Institute for Mathematical Science

KONKUK UNIVERSITY

1 HWAYANG-DONG

GWANGJIN-GU, SEOUL 143-701

REPUBLIC OF KOREA

kszoo@postech.ac.kr 


\title{
PACIFIC JOURNAL OF MATHEMATICS
}

\author{
msp.org/pjm
}

Founded in 1951 by E. F. Beckenbach (1906-1982) and F. Wolf (1904-1989)

\section{EDITORS}

V. S. Varadarajan (Managing Editor)

Department of Mathematics

University of California

Los Angeles, CA 90095-1555

pacific@math.ucla.edu

Paul Balmer

Department of Mathematics

University of California

Los Angeles, CA 90095-1555

balmer@math.ucla.edu

Daryl Cooper

Department of Mathematics

University of California

Santa Barbara, CA 93106-3080 cooper@math.ucsb.edu

Jiang-Hua $\mathrm{Lu}$

Department of Mathematics

Pokfulam Rd., Hong Kong jhlu@maths.hku.hk
The University of Hong Kong

Don Blasius

Department of Mathematics University of California

Los Angeles, CA 90095-1555

blasius@math.ucla.edu

Robert Finn

Department of Mathematics Stanford University

Stanford, CA 94305-2125

finn@math.stanford.edu

Sorin Popa

Department of Mathematics

University of California

Los Angeles, CA 90095-1555 popa@math.ucla.edu

Paul Yang

Department of Mathematics Princeton University

Princeton NJ 08544-1000

yang@math.princeton.edu

\section{PRODUCTION}

Silvio Levy, Scientific Editor, production@msp.org

\section{SUPPORTING INSTITUTIONS}

ACADEMIA SINICA, TAIPEI

CALIFORNIA INST. OF TECHNOLOGY

INST. DE MATEMÁTICA PURA E APLICADA

KEIO UNIVERSITY

MATH. SCIENCES RESEARCH INSTITUTE

NEW MEXICO STATE UNIV.

OREGON STATE UNIV.

\author{
STANFORD UNIVERSITY \\ UNIV. OF BRITISH COLUMBIA \\ UNIV. OF CALIFORNIA, BERKELEY \\ UNIV. OF CALIFORNIA, DAVIS \\ UNIV. OF CALIFORNIA, LOS ANGELES \\ UNIV. OF CALIFORNIA, RIVERSIDE \\ UNIV. OF CALIFORNIA, SAN DIEGO \\ UNIV. OF CALIF., SANTA BARBARA
}

\author{
Vyjayanthi Chari \\ Department of Mathematics \\ University of California \\ Riverside, CA 92521-0135 \\ chari@math.ucr.edu \\ Kefeng Liu \\ Department of Mathematics \\ University of California \\ Los Angeles, CA 90095-1555 \\ liu@math.ucla.edu \\ Jie Qing \\ Department of Mathematics \\ University of California \\ Santa Cruz, CA 95064 \\ qing@cats.ucsc.edu
}

These supporting institutions contribute to the cost of publication of this Journal, but they are not owners or publishers and have no responsibility for its contents or policies.

See inside back cover or msp.org/pjm for submission instructions.

The subscription price for 2013 is US \$400/year for the electronic version, and \$485/year for print and electronic.

Subscriptions, requests for back issues and changes of subscribers address should be sent to Pacific Journal of Mathematics, P.O. Box 4163, Berkeley, CA 94704-0163, U.S.A. The Pacific Journal of Mathematics is indexed by Mathematical Reviews, Zentralblatt MATH, PASCAL CNRS Index, Referativnyi Zhurnal, Current Mathematical Publications and the Science Citation Index.

The Pacific Journal of Mathematics (ISSN 0030-8730) at the University of California, c/o Department of Mathematics, 798 Evans Hall \#3840, Berkeley, CA 94720-3840, is published twelve times a year. Periodical rate postage paid at Berkeley, CA 94704, and additional mailing offices. POSTMASTER: send address changes to Pacific Journal of Mathematics, P.O. Box 4163, Berkeley, CA 94704-0163.

PJM peer review and production are managed by EditFLOW ${ }^{\circledR}$ from Mathematical Sciences Publishers.

\section{PUBLISHED BY}

mathematical sciences publishers

nonprofit scientific publishing

http://msp.org/

(C) 2013 Mathematical Sciences Publishers 


\section{PACIFIC JOURNAL OF MATHEMATICS}

Volume $266 \quad$ No. $1 \quad$ November 2013

Multiplicity of solutions to the Yamabe problem on collapsing $\quad 1$ Riemannian submersions

Renato G. Bettiol and Paolo Piccione

Rank gradient of small covers

DARLAN GIRÃO

Nonrationality of nodal quartic threefolds

KYUSIK HONG

Supertropical linear algebra

ZUR IZHAKIAN, MANFRED KNEBUSCH and LOUIS ROWEN

Isometry groups among topological groups

PIOTR NIEMIEC

Singularities and Liouville theorems for some special conformal

Hessian equations

QIANZHONG OU

Attaching handles to Delaunay nodoids

FRANK PACARD and HAROLD ROSENBERG

Some new canonical forms for polynomials

BRUCE REZNICK

Applications of the deformation formula of holomorphic one-forms 